\title{
ON CONVEXITY OF SIMPLE CLOSED FRONTALS
}

\author{
TOMONORi Fukunaga AND Masatomo TAKahashi
}

\begin{abstract}
We study convexity of simple closed frontals of Legendre curves in the Euclidean plane by using the curvature of Legendre curves. We show that for a Legendre curve, the simple closed frontal under conditions is convex if and only if the sign of both functions of the curvature of the Legendre curve does not change. We also give some examples of convex simple closed frontals.
\end{abstract}

\section{Introduction and main result}

In the classical differential geometry of regular curves, we can analyze global properties of curves, such as convexity, width and rotation number by using the curvature (cf. [4], [5]). One of well-known result is a characterization of convexity of simple closed regular curves by using the curvature (cf. [5]):

THEOREM 1.1. A simple closed regular curve is convex if and only if its curvature $\kappa$ has a constant sign, that is, $\kappa$ is either always non-positive or always non-negative.

When we consider singular curves, the above theorem does not hold, that is, there is a simple closed singular curve such that the curvature is always non-positive except singular points, but the curve is not convex (the curve divided by a tangent line). For example, let $\gamma:[0,2 \pi] \rightarrow \mathbf{R}^{2}$ be the astroid $\gamma(t)=$ $\left(\cos ^{3} t, \sin ^{3} t\right)$. The curvature $\kappa$ of $\gamma$ is given by $\kappa(t)=-2 /(3|\sin 2 t|)$ except four singular points and diverges to $-\infty$ at each singular points. Hence, $\kappa$ has the constant sign. However, this curve is not convex, see Figure 1.

In the present paper, we give a characterization of convexity for a special class of singular curves called frontals by using the curvature of Legendre curves which has introduced in [3].

Let $I$ be an interval. We say that $(\gamma, v): I \rightarrow \mathbf{R}^{2} \times S^{1}$ is a Legendre curve if $(\gamma(t), v(t))^{*} \alpha=0$ for all $t \in I$, where $\alpha$ is the canonical contact 1 -form on the unit tangent bundle $T_{1} \mathbf{R}^{2}=\mathbf{R}^{2} \times S^{1}=\left\{(x, y, a, b) \in \mathbf{R}^{4} \mid a^{2}+b^{2}=1\right\}$ defined by $\alpha=$

2010 Mathematics Subject Classification. 58K05, 53A04, 57R45.

Key words and phrases. Legendre curve, frontal, convex, simple closed.

Received August 5, 2015; revised October 27, 2015. 


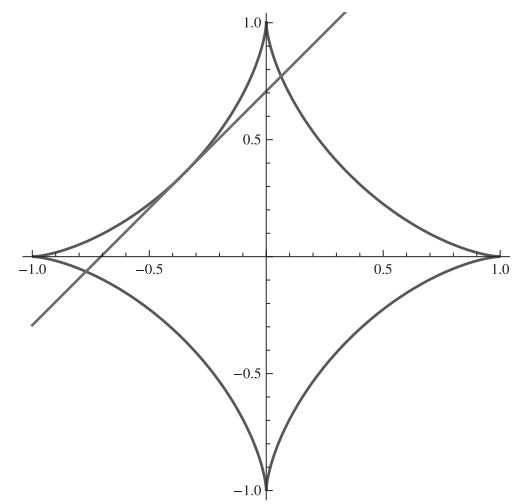

FIGURE 1. The tangent line at $t=3 \pi / 4$ of the astroid $\gamma(t)=\left(\cos ^{3} t, \sin ^{3} t\right)$ divide the curve.

$a d x+b d y$ (cf. [1], [2]). This condition is equivalent to $\dot{\gamma}(t) \cdot v(t)=0$ for all $t \in I$, where - is the Euclidean inner product on $\mathbf{R}^{2}$. We say that $\gamma: I \rightarrow \mathbf{R}^{2}$ is $a$ frontal if there exists a smooth mapping $v: I \rightarrow S^{1}$ such that $(\gamma, v)$ is a Legendre curve. Note that, if a plane curve $\gamma: I \rightarrow \mathbf{R}^{2}$ is not infinitely flat at $t_{0} \in I$, then $\gamma$ is a frontal around $t_{0}$. See [3] for more details.

Let $(\gamma, v): I \rightarrow \mathbf{R}^{2} \times S^{1}$ be a Legendre curve. If the frontal $\gamma$ is a regular curve around a point $t_{0}$, then we have the Frenet frame along $\gamma$. On the other hand, if the frontal $\gamma$ is singular at a point $t_{0}$, then we can not define the Frenet frame. However, $v$ is always defined even if $t_{0}$ is a singular point of $\gamma$. Therefore, we have a frame along the frontal $\gamma$ as follows. We put on $\boldsymbol{\mu}(t)=J(v(t))$, where $J$ is the anti-clockwise $\pi / 2$ rotation on $\mathbf{R}^{2}$. We call the pair $\{v(t), \boldsymbol{\mu}(t)\}$ a moving frame along the frontal $\gamma(t)$ in $\mathbf{R}^{2}$ and we have the Frenet formula of the frontal (or, the Legendre curve) which is given by

$$
\left(\begin{array}{c}
\dot{v}(t) \\
\dot{\boldsymbol{\mu}}(t)
\end{array}\right)=\left(\begin{array}{cc}
0 & \ell(t) \\
-\ell(t) & 0
\end{array}\right)\left(\begin{array}{c}
v(t) \\
\boldsymbol{\mu}(t)
\end{array}\right),
$$

where $\ell(t)=\dot{v}(t) \cdot \boldsymbol{\mu}(t)$. Moreover, there exists a smooth function $\beta(t)$ such that

$$
\dot{\gamma}(t)=\beta(t) \boldsymbol{\mu}(t) .
$$

The pair $(\ell, \beta)$ is an important invariant of Legendre curves (or, frontals). We call the pair $(\ell(t), \beta(t))$ the curvature of the Legendre curve (with respect to the parameter $t$ ).

Remark 1.2. Let $(\gamma, v): I \rightarrow \mathbf{R}^{2} \times S^{1}$ and $(\bar{\gamma}, \bar{v}): \bar{I} \rightarrow \mathbf{R}^{2} \times S^{1}$ be Legendre curves whose curvatures of Legendre curves are $(\ell, \beta)$ and $(\bar{\ell}, \bar{\beta})$ respectively. Suppose that $(\gamma, v)$ and $(\bar{\gamma}, \bar{v})$ are parametrically equivalent via the change of parameter $t: \bar{I} \rightarrow I ; u \mapsto t(u)$ with $\dot{t}(u)>0$, that is, $(\bar{\gamma}(u), \bar{v}(u))=(\gamma(t(u)), v(t(u)))$ 
for all $u \in \bar{I}$. Then, we have

$$
\bar{\ell}(u)=\ell(t(u)) \dot{t}(u), \quad \bar{\beta}(u)=\beta(t(u)) \dot{t}(u) .
$$

Hence the curvature of the Legendre curve is depended on a choice of a parametrization.

Definition 1.3. Let $(\gamma, v)$ and $(\tilde{\gamma}, \tilde{v}): I \rightarrow \mathbf{R}^{2} \times S^{1}$ be Legendre curves. We say that $(\gamma, v)$ and $(\tilde{\gamma}, \tilde{v})$ are congruent as Legendre curves if there exists a congruence $C$ on $\mathbf{R}^{2}$ such that $\tilde{\gamma}(t)=C(\gamma(t))=A(\gamma(t))+\boldsymbol{b}$ and $\tilde{v}(t)=A(v(t))$ for all $t \in I$, where $C$ is given by the rotation $A$ and the translation $\boldsymbol{b}$ on $\mathbf{R}^{2}$.

Then we have the following theorems.

Theorem 1.4 (The Existence Theorem, [3]). Let $(\ell, \beta): I \rightarrow \mathbf{R}^{2}$ be a smooth mapping. There exists a Legendre curve $(\gamma, v): I \rightarrow \mathbf{R}^{2} \times S^{1}$ whose associated curvature of the Legendre curve is $(\ell, \beta)$.

THEOREM 1.5 (The Uniqueness Theorem, [3]). Let $(\gamma, v)$ and $(\tilde{\gamma}, \tilde{v}): I \underset{\sim}{\rightarrow}$ $\mathbf{R}^{2} \times S^{1}$ be Legendre curves whose curvatures of Legendre curves $(\ell, \beta)$ and $(\tilde{\ell}, \tilde{\beta})$ coincide. Then $(\gamma, v)$ and $(\tilde{\gamma}, \tilde{v})$ are congruent as Legendre curves.

For $n \in \mathbf{N} \cup\{0\}$, we say that a Legendre curve $(\gamma, v):[a, b] \rightarrow \mathbf{R}^{2} \times S^{1}$ is $C^{n}$-closed if $\left(\gamma^{(k)}(a), v^{(k)}(a)\right)=\left(\gamma^{(k)}(b), v^{(k)}(b)\right)$ for all $k \in\{0, \ldots, n\}$, where $\gamma^{(k)}(a), v^{(k)}(a), \gamma^{(k)}(b)$ and $v^{(k)}(b)$ mean one-sided $k$-th differential. Similarly, we say that a Legendre curve $(\gamma, v):[a, b] \rightarrow \mathbf{R}^{2} \times S^{1}$ is $C^{\infty}$-closed if $\left(\gamma^{(k)}(a), v^{(k)}(a)\right)=\left(\gamma^{(k)}(b), v^{(k)}(b)\right)$ for all $k \in \mathbf{N} \cup\{0\}$. In this paper, we say that $(\gamma, v)$ is a closed Legendre curve, if the curve is at least $C^{1}$-closed. Note that if $(\gamma, v)$ is a closed Legendre curve, the domain of the curve can be extended from $[a, b]$ to $\mathbf{R}$ so that $(\gamma, v)([a, b])=(\gamma, v)(\mathbf{R})$ and the extended map $(\gamma, v): \mathbf{R} \rightarrow$ $\mathbf{R}^{2} \times S^{1}$ is at least $C^{1}$ map. Moreover, a frontal $\gamma:[a, b] \rightarrow \mathbf{R}^{2}$ is simple closed if for $t_{1}<t_{2}$, we have $\gamma\left(t_{1}\right)=\gamma\left(t_{2}\right)$ if and only if $t_{1}=a$ and $t_{2}=b$.

We define a convex frontal in the Euclidean plane. From now on, $I$ is a closed interval. Let $(\gamma, v): I \rightarrow \mathbf{R}^{2} \times S^{1}$ be a Legendre curve. We denote the tangent line at $t$ of $\gamma(t)$ by $L_{t}$, that is, $L_{t}=\{\lambda \boldsymbol{\mu}(t)+\gamma(t) \mid \lambda \in \mathbf{R}\}$. Any tangent line $L_{t}$ divides $\mathbf{R}^{2}$ into two half-planes $H_{+}(t)$ and $H_{-}(t)$ such that $H_{+}(t) \cup$ $H_{-}(t)=\mathbf{R}^{2}$ and $H_{+}(t) \cap H_{-}(t)=L_{t}$. By using $v$, the half-planes $H_{+}(t)$ and $H_{-}(t)$ are presented by $H_{+}(t)=\left\{\boldsymbol{x} \in \mathbf{R}^{2} \mid(\boldsymbol{x}-\gamma(t)) \cdot v(t) \geq 0\right\}$ and $H_{-}(t)=$ $\left\{\boldsymbol{x} \in \mathbf{R}^{2} \mid(\boldsymbol{x}-\gamma(t)) \cdot v(t) \leq 0\right\}$. For a Legendre curve $(\gamma, v): I \rightarrow \mathbf{R}^{2} \times S^{1}$, we say that $\gamma$ is a convex frontal (or, $(\gamma, v)$ is a convex Legendre curve) if $\gamma(I) \subset H_{+}(t)$ for any $t \in I$ or $\gamma(I) \subset H_{-}(t)$ for any $t \in \mathbf{R}$. Note that if $\gamma$ is a regular curve, then $\boldsymbol{\mu}(t)$ is equal to the unit tangent vector of $\gamma$ at $\gamma(t)$ up to sign. Therefore, $\gamma$ is convex as a frontal if and only if $\gamma$ is convex curve as a regular curve (cf. [5]).

By definition, convexity of a Legendre curve is preserved under a congruence as Legendre curves. Moreover, if $(\gamma, v): I \rightarrow \mathbf{R}^{2} \times S^{1}$ is a convex Legendre 
curve, then $(\gamma \circ u, v \circ u): \bar{I} \rightarrow \mathbf{R}^{2} \times S^{1}$ is also convex for a change of parameter $u: \bar{I} \rightarrow I$ and any smooth function $u: \bar{I} \rightarrow I$ as well.

The main result of this paper is stated as follows:

THEOREM 1.6. Let $(\gamma, v): I \rightarrow \mathbf{R}^{2} \times S^{1}$ be a closed Legendre curve with the curvature $(\ell, \beta)$ which the frontal $\gamma$ is simple closed. Suppose that zeros of $\ell$ and of $\beta$ are isolated points. Then the frontal $\gamma$ is convex if and only if the curvature satisfy one of the following condition:

(i) Both of $\ell(t)$ and $\beta(t)$ are always non-negative,

(ii) $\ell(t)$ is always non-negative and $\beta(t)$ is always non-positive,

(iii) Both of $\ell(t)$ and $\beta(t)$ are always non-positive,

(iv) $\ell(t)$ is always non-positive and $\beta(t)$ is always non-negative.

We prove this theorem in Section 2. Moreover, we give examples of convex simple closed frontals in Section 3.

Acknowledgement. The authors would like to thank the referee for helpful comments to improve the original manuscript. The first author was partially supported by JSPS KAKENHI Grant Number 15K17457 and the second author was partially supported by JSPS KAKENHI Grant Number 26400078.

\section{Proof of the main result}

Let $(\gamma, v): I \rightarrow \mathbf{R}^{2} \times S^{1}$ be a closed Legendre curve with the curvature $(\ell, \beta)$. In this paper, we assume that zeros of $\ell$ and $\beta$ are isolated points. First, we prove the following Lemma.

LEMma 2.1. Let $(\gamma, v): I \rightarrow \mathbf{R}^{2} \times S^{1}$ be a closed Legendre curve. If the sign of $\ell$ or the sign of $\beta$ change, then the frontal $\gamma$ is not convex.

Proof. Let $t_{0} \in I$ be a point such that the sign of $\ell$ or the sign of $\beta$ change, that is, locally $\ell(t)>0$ (respectively, $\ell(t)<0$ ) if $t<t_{0}$ and $\ell(t)<0$ (respectively, $\ell(t)>0$ ) if $t>t_{0}$, or $\beta(t)>0$ (respectively, $\beta(t)<0$ ) if $t<t_{0}$ and $\beta(t)<0$ (respectively, $\beta(t)>0$ ) if $t>t_{0}$. Since convexity of the frontal does not change by a congruence of Legendre curves, we may assume $\gamma\left(t_{0}\right)$ is the origin of the Euclidean plane without loss of generality.

If the sign of $\gamma(t) \cdot v\left(t_{0}\right)$ changes around $t_{0}$, then $L_{t_{0}}$ divide the frontal $\gamma$. To prove that the frontal $\gamma$ is not convex, we show that the sign of $\gamma(t) \cdot v\left(t_{0}\right)$ changes around $t_{0}$.

By the definition of $\beta$, we have

$$
\frac{d}{d t}\left(\gamma(t) \cdot v\left(t_{0}\right)\right)=\beta(t) \boldsymbol{\mu}(t) \cdot v\left(t_{0}\right) .
$$


Since $\|\boldsymbol{\mu}(t)\|=\left\|v\left(t_{0}\right)\right\|=1$, there is a smooth function $\varphi$ such that $\boldsymbol{\mu}(t) \cdot v\left(t_{0}\right)=$ $\cos \varphi(t)$. Moreover, by the definition of $\ell$, we have

$$
-\dot{\varphi}(t) \sin \varphi(t)=\frac{d}{d t}\left(\boldsymbol{\mu}(t) \cdot v\left(t_{0}\right)\right)=-\ell(t) v(t) \cdot v\left(t_{0}\right) .
$$

Since $\cos \varphi\left(t_{0}\right)=\boldsymbol{\mu}\left(t_{0}\right) \cdot v\left(t_{0}\right)=0$, we have $\sin \varphi\left(t_{0}\right)= \pm 1$. Substitute $t_{0}$ for the equation (1), we obtain

$$
\dot{\varphi}\left(t_{0}\right)= \pm \ell\left(t_{0}\right) .
$$

First, we consider the case of the sign of $\ell$ changes and the sign of $\beta$ does not change around $t_{0}$. In this case, $\dot{\varphi}\left(t_{0}\right)=0$ by (2). Since $v(t) \cdot v\left(t_{0}\right)$ is a continuous function and $v\left(t_{0}\right) \cdot v\left(t_{0}\right)=1$, we have $v(t) \cdot v\left(t_{0}\right)>0$ around $t_{0}$. By the equation (1), the sign of $\dot{\varphi}(t)$ changes around $t_{0}$. This conclude that the sign of $\cos \varphi(t)$ does not change around $t_{0}$. Hence $\boldsymbol{\mu}(t) \cdot v\left(t_{0}\right) \geq 0$ or $\boldsymbol{\mu}(t) \cdot v\left(t_{0}\right) \leq 0$ around $t_{0}$. Moreover, since the sign of $\beta$ does not change around $t_{0}$, we obtain $(d / d t)\left(\gamma(t) \cdot v\left(t_{0}\right)\right) \geq 0$ or $(d / d t)\left(\gamma(t) \cdot v\left(t_{0}\right)\right) \leq 0$, that is, $\gamma(t) \cdot v\left(t_{0}\right)$ is a monotone function around $t_{0}$. By the assumption $\gamma\left(t_{0}\right)=0$, we have $\gamma\left(t_{0}\right) \cdot v\left(t_{0}\right)=0$ and the sign of $\gamma(t) \cdot v\left(t_{0}\right)$ changes around $t_{0}$. Therefore, $\gamma$ is not convex.

Second, we consider the case of the sign of $\beta$ changes and the sign of $\ell$ does not change around $t_{0}$. By the equation (1), the sign of $\dot{\varphi}(t)$ does not change around $t_{0}$, that is, $\dot{\varphi}(t)$ is a monotone function around $t_{0}$. This conclude that the sign of $\cos \varphi(t)$ changes around $t_{0}$. Hence the sign of $\boldsymbol{\mu}(t) \cdot v\left(t_{0}\right)$ changes around $t_{0}$. It follows that the sign of $(d / d t)\left(\gamma(t) \cdot v\left(t_{0}\right)\right)$ does not change around $t_{0}$. By the same argument of the first case, $\gamma$ is not convex.

Finally, we consider the case of the signs of $\ell$ and $\beta$ change around $t_{0}$ simultaneously. We prove by a contradiction. Assume that the frontal $\gamma$ is a convex curve. Since the signs of $\ell$ and $\beta$ change around $t_{0}$, similar to the first and second cases, the sign of $(d / d t)\left(\gamma(t) \cdot v\left(t_{0}\right)\right)$ changes around $t_{0}$. Moreover,

$$
\frac{d}{d t}\left(\gamma(t) \cdot \boldsymbol{\mu}\left(t_{0}\right)\right)=\beta(t) \boldsymbol{\mu}(t) \cdot \boldsymbol{\mu}\left(t_{0}\right) .
$$

Since the sign of $\beta$ changes and $\boldsymbol{\mu}(t) \cdot \boldsymbol{\mu}\left(t_{0}\right)>0$ around $t_{0}$, the sign of $(d / d t)\left(\gamma(t) \cdot \boldsymbol{\mu}\left(t_{0}\right)\right)$ also changes around $t_{0}$. Hence, $\gamma$ is contained in a quadrant of the $\left\{v\left(t_{0}\right), \boldsymbol{\mu}\left(t_{0}\right)\right\}$-plane around $t_{0}$, namely, $\gamma$ is contained one of the followings: the first quadrant $\left\{a v\left(t_{0}\right)+b \boldsymbol{\mu}\left(t_{0}\right) \mid a, b \geq 0\right\}$, the second quadrant $\left\{a v\left(t_{0}\right)+b \boldsymbol{\mu}\left(t_{0}\right) \mid a \leq 0, b \geq 0\right\}$, the third quadrant $\left\{a v\left(t_{0}\right)+b \boldsymbol{\mu}\left(t_{0}\right) \mid a \leq 0, b \leq 0\right\}$ or the fourth quadrant $\left\{a v\left(t_{0}\right)+b \boldsymbol{\mu}\left(t_{0}\right) \mid a \geq 0, b \leq 0\right\}$.

Since $(d / d t)\left(\boldsymbol{\mu}(t) \cdot v\left(t_{0}\right)\right)=-\ell(t) v(t) \cdot v\left(t_{0}\right)$, the sign of $(d / d t)\left(\boldsymbol{\mu}(t) \cdot v\left(t_{0}\right)\right)$ changes around $t_{0}$ and $(d / d t)\left(\boldsymbol{\mu}\left(t_{0}\right) \cdot v\left(t_{0}\right)\right)=0$. Therefore, $t_{0}$ is a local maximal or local minimal point of $\boldsymbol{\mu}(t) \cdot v\left(t_{0}\right)$. It follows that for any $\varepsilon>0$, there exist $t_{1} \in\left(t_{0}-\varepsilon, t_{0}\right)$ and $t_{2} \in\left(t_{0}, t_{0}+\varepsilon\right)$ such that

$$
\boldsymbol{\mu}\left(t_{1}\right) \cdot v\left(t_{0}\right)=\boldsymbol{\mu}\left(t_{2}\right) \cdot v\left(t_{0}\right)
$$

Since $\left\{v\left(t_{0}\right), \boldsymbol{\mu}\left(t_{0}\right)\right\}$ is a basis on $\mathbf{R}^{2}$, there exist smooth functions $\lambda$ and $\eta$ such that $\boldsymbol{\mu}(t)=\lambda(t) v\left(t_{0}\right)+\eta(t) \boldsymbol{\mu}\left(t_{0}\right)$. By the equation (3), we have $\lambda\left(t_{1}\right)=\lambda\left(t_{2}\right)$. 
Moreover, since $\boldsymbol{\mu}\left(t_{1}\right)$ and $\boldsymbol{\mu}\left(t_{2}\right)$ are unit vectors, we have $\lambda^{2}\left(t_{1}\right)+\eta^{2}\left(t_{1}\right)=$ $\lambda^{2}\left(t_{2}\right)+\eta^{2}\left(t_{2}\right)$. Hence, $\eta\left(t_{1}\right)= \pm \eta\left(t_{2}\right)$. By the definition of $\eta$, we have $\eta\left(t_{0}\right)=1$. It follows that both $\eta\left(t_{1}\right)$ and $\eta\left(t_{2}\right)$ are positive. Thus $\eta\left(t_{1}\right)=\eta\left(t_{2}\right)$ and hence $\boldsymbol{\mu}\left(t_{1}\right)=\boldsymbol{\mu}\left(t_{2}\right)$. It follows that $L_{t_{1}}$ is parallel to $L_{t_{2}}$. Since the zeros of $\beta$ are isolated, by changing of $\varepsilon$ if necessary, we can assume $\gamma\left(t_{1}\right) \neq \gamma\left(t_{2}\right)$.

Now suppose that $L_{t_{1}}=L_{t_{2}}$. Since we assume $\gamma$ is a convex curve, the curve lies on one-side of $L_{t_{1}}\left(=L_{t_{2}}\right)$ and tangent at $\gamma\left(t_{1}\right)$ and $\gamma\left(t_{2}\right)$. Since zeros of $\ell$ are isolated points, $L_{t_{1}}$ is a double tangent line of $\gamma$. It follows that, there exists a point $t_{3}$ around $t_{1}$ or $t_{2}$ such that $L_{t_{3}}$ divide $\gamma(I)$. This contradict to convexity of $\gamma$. Hence, we have $L_{t_{1}} \neq L_{t_{2}}$.

On the other hand, when $L_{t_{1}} \neq L_{t_{2}}$, we obtain $\gamma(I) \subset H_{+}\left(t_{1}\right) \cap H_{+}\left(t_{2}\right)$ or $\gamma(I) \subset H_{-}\left(t_{1}\right) \cap H_{-}\left(t_{2}\right)$ by the definition of the convex. The tangent line $L_{t_{1}}$ or $L_{t_{2}}$ divide $\gamma(I)$, since $\gamma$ lies on a same side of half-planes which are divided by the parallel tangent lines $L_{t_{1}}$ and $L_{t_{2}}$. This contradict to convexity of $\gamma$. Therefore, $\gamma$ is not convex.

In the rest of this section we prove that if the signs of $\ell$ and $\beta$ do not change, then the simple closed frontal $\gamma$ is convex. In order to prove this claim, we prepare lemmas and notations.

Lemma 2.2. Let $(\gamma, v): I \rightarrow \mathbf{R}^{2} \times S^{1}$ be a Legendre curve with the curvature $(\ell, \beta)$. If $\beta(t) \geq 0$ for all $t \in I$ or $\beta(t) \leq 0$ for all $t \in I$, then there is the smooth map $\Phi(\gamma, v): I \rightarrow S^{1}$ such that $\Phi(\gamma, v)=\dot{\gamma} /\|\dot{\gamma}\|$ on $I \backslash \mathscr{Z}_{\beta}$, where $\mathscr{Z}_{\beta}=\{t \in I \mid$ $\beta(t)=0\}$.

Proof. By the definition of $\beta$, we obtain

$$
\frac{\dot{\gamma}(t)}{\|\dot{\gamma}(t)\|}=\frac{\beta(t)}{|\beta(t)|} \frac{\boldsymbol{\mu}(t)}{\|\boldsymbol{\mu}(t)\|}=\frac{\beta(t)}{|\beta(t)|} \boldsymbol{\mu}(t)=\operatorname{sign}(\beta(t)) \boldsymbol{\mu}(t)
$$

on $I \backslash \mathscr{Z}_{\beta}$. Here we define $\operatorname{sign}(\beta(t))$ by 1 if $\beta(t) \geq 0$ for all $t \in I$ and -1 if $\beta(t) \leq 0$ for all $t \in I$. Since $\operatorname{sign}(\beta(t))$ is a constant, the map $\Phi(\gamma, v)(t)=$ $\operatorname{sign}(\beta(t)) \boldsymbol{\mu}(t)$ is a smooth map on $I$. This is the required map.

We denote the set of closed Legendre curves $(\gamma, v)$ with $\beta(t) \geq 0$ (respectively, $\beta(t) \leq 0)$ for all $t \in I$ by $\mathscr{R}_{+}$(respectively, $\mathscr{R}_{-}$), and $\mathscr{R}_{+} \cup \mathscr{R}_{-}$by $\mathscr{R}$. For a Legendre curve $(\gamma, v) \in \mathscr{R}$, we define a smooth function $\theta: I \rightarrow \mathbf{R}$ such that $\Phi(\gamma, v)(t)=(\cos \theta(t), \sin \theta(t))$ by Lemma 2.2. Same as the case of regular curves, we call the mapping degree of $\Phi(\gamma, v)$ the rotation index of the Legendre curve $(\gamma, v) \in \mathscr{R}$.

LEMmA 2.3. If $(\gamma, v) \in \mathscr{R}$, then $\ell(t)=-\operatorname{sign}(\beta(t)) \dot{\theta}(t)$.

Proof. Suppose $(\gamma, v) \in \mathscr{R}$, we have

$$
\boldsymbol{\mu}(t)=\operatorname{sign}(\beta(t)) \Phi(\gamma, v)(t)=\operatorname{sign}(\beta(t))(\cos \theta(t), \sin \theta(t))
$$


and

$$
v(t)=J^{-1}(\boldsymbol{\mu}(t))=\operatorname{sign}(\beta(t))(\sin \theta(t),-\cos \theta(t)) .
$$

Then $\dot{\boldsymbol{\mu}}(t)=-\operatorname{sign}(\beta(t)) \dot{\theta}(t) v(t)$. By the definition of $\ell$, we obtain $\ell(t)=$ $-\operatorname{sign}(\beta(t)) \dot{\theta}(t)$.

Lemma 2.4. Let $(\gamma, v): I \rightarrow \mathbf{R}^{2} \times S^{1}$ be a Legendre curve with $(\gamma, v) \in \mathscr{R}$. Then there is a point $t_{0} \in I$ such that $\gamma(I)$ lies entirely to one side of $L_{t_{0}}$.

Proof. Let $(x, y)$ be a coordinate on $\mathbf{R}^{2}$ and let $\gamma\left(t_{0}\right)=p$ be a point which has maximum height, that is, $y$-coordinate is maximum in $\gamma(I)$. Without loss of generality, we may assume $p$ is the origin of the Euclidean plane and $(\gamma, v) \in \mathscr{R}_{+}$. Since the sign of $\beta$ does not change and $\boldsymbol{\mu}\left(t_{0}\right) \cdot \boldsymbol{\mu}\left(t_{0}\right)=1$, we have

$$
\frac{d}{d t}\left(\gamma(t) \cdot \boldsymbol{\mu}\left(t_{0}\right)\right)=\beta(t) \boldsymbol{\mu}(t) \cdot \boldsymbol{\mu}\left(t_{0}\right) \geq 0
$$

around $t_{0}$. If the sign of $(d / d t)\left(\gamma(t) \cdot v\left(t_{0}\right)\right)$ does not change around $t_{0}$, then $\gamma$ through the origin from the second quadrant to the fourth quadrant or the third quadrant to the first quadrant in the $\left\{v\left(t_{0}\right), \boldsymbol{\mu}\left(t_{0}\right)\right\}$-plane. This contradicts the fact $p$ has maximum height. Hence, the sign of $(d / d t)\left(\gamma(t) \cdot v\left(t_{0}\right)\right)$ changes around $t_{0}$. This means $\gamma$ lies on the under half-plane divided by $L_{t_{0}}$. Moreover, if $L_{t_{0}}$ does not coincides with the $x$-axis, this contradicts the fact $p$ has maximum height. Therefore, $L_{t_{0}}$ coincides with the $x$-axis. By the above, $L_{t_{0}}$ coincides with the $x$-axis and $\gamma(I)$ lies on under the $x$-axis. Therefore, $t_{0}$ is the required point.

The rest of the proof is similar to the case of regular curves (see [5]).

LEMma 2.5. Let $(\gamma, v): I \rightarrow \mathbf{R}^{2} \times S^{1}$ be a Legendre curve which the frontal $\gamma$ is simple closed and $(\gamma, v) \in \mathscr{R}$. Then the rotation index of the Legendre curve $(\gamma, v)$ is equal to \pm 1 .

Proof. Let $p=\gamma\left(t_{0}\right)$ be a point on $\gamma(I)$ with the property that $\gamma(I)$ lies entirely to one side of $L_{t_{0}}$. Such a point $t_{0}$ always exists by Lemma 2.4 .

Now we set $I=[0, L]$. Consider the triangular region $\mathscr{T}:=\left\{\left(t_{1}, t_{2}\right) \in \mathbf{R}^{2} \mid\right.$ $\left.0 \leq t_{1} \leq t_{2} \leq L\right\}$. By a reparametrization of $(\gamma, v)$, we may assume $t_{0}=0$, that is, $\gamma(0)=p$. We define a map $\Sigma: \mathscr{T} \rightarrow S^{1}$ by

$$
\Sigma\left(t_{1}, t_{2}\right)=\left\{\begin{array}{l}
\Phi(\gamma, v)(t) \quad\left(\text { if } t_{1}=t_{2}=t\right) \\
-\Phi(\gamma, v)(0) \quad\left(\text { if } t_{1}=0 \text { and } t_{2}=L\right), \\
\frac{\gamma\left(t_{2}\right)-\gamma\left(t_{1}\right)}{\left\|\gamma\left(t_{2}\right)-\gamma\left(t_{1}\right)\right\|} \quad \text { (otherwise) }
\end{array}\right.
$$

By the assumptions and Lemma 2.2, the map $\Sigma$ is continuous. Let $A=(0,0)$, $B=(0, L)$ and $C=(L, L)$. Since the restriction of $\Sigma$ to the segment $A C$ is 
equal to $\Phi(\gamma, v)$, the mapping degree of this map is equal to the rotation index of $(\gamma, v)$.

Moreover, consider the restriction of $\Sigma$ to the segment $A B \cup B C$. Set the angle from $\Phi(\gamma, v)(0)$ to $-\Phi(\gamma, v)(0)$ is equal to $\pi$. Since

$$
\left.\Sigma\right|_{A B}\left(t_{1}, t_{2}\right)=\Sigma(0, t)=(\gamma(t)-\gamma(0)) /\|\gamma(t)-\gamma(0)\|,
$$

$\left.\Sigma\right|_{A B}$ covers one half of $S^{1}$. Similarly,

$$
\left.\Sigma\right|_{B C}\left(t_{1}, t_{2}\right)=\Sigma(t, L)=(\gamma(L)-\gamma(t)) /\|\gamma(L)-\gamma(t)\|,
$$

$\left.\Sigma\right|_{B C}$ covers other half of $S^{1}$. Hence, the mapping degree of the restriction of $\Sigma$ to the segment $A B \cup B C$ is equal to \pm 1 (the sign depends on an orientation of $\gamma$ ).

Note that the restriction of $\Sigma$ to the segment $A B \cup B C$ is homotopic to the restriction of $\Sigma$ to the segment $A C$, that is, $\Phi(\gamma, v)$. Because the mapping degree is preserved under homotopy, the mapping degree of $\Phi(\gamma, v)$ is equal to \pm 1 . Therefore, the rotation index of the Legendre curve $(\gamma, v)$ is equal to \pm 1 .

The following lemma is the sufficient part of the main theorem.

Lemma 2.6. Let $(\gamma, v): I \rightarrow \mathbf{R}^{2} \times S^{1}$ be a closed Legendre curve which the frontal $\gamma$ is simple closed. If the signs of $\ell$ and $\beta$ do not change, then the frontal $\gamma$ is convex.

Proof. By Lemma 2.3, the sign of $\ell(t)$ does not change if and only if $\theta$ is a monotone function on $I$. Suppose that $\gamma$ is not convex. There is a point $\gamma\left(s_{0}\right) \in \gamma(I)$ such that $L_{s_{0}}$ divide $\gamma$ to $\gamma_{1} \subset H_{+}\left(s_{0}\right)$ and $\gamma_{2} \subset H_{-}\left(s_{0}\right)$. By the intermediate value theorem on $\theta$, there are two points $\gamma\left(s_{1}\right) \in \gamma_{1}$ and $\gamma\left(s_{2}\right) \in \gamma_{2}$ such that $\theta\left(s_{1}\right)=\theta\left(s_{0}\right)$ up to $\pm n \pi, L_{s_{1}} \neq L_{s_{2}}, \theta\left(s_{2}\right)=\theta\left(s_{0}\right)$ up to $\pm n \pi$ and $L_{s_{2}} \neq L_{s_{0}}, L_{s_{1}}$ for some integer $n$.

Two of the three points $\gamma\left(s_{0}\right), \gamma\left(s_{1}\right)$ and $\gamma\left(s_{2}\right)$ must have tangents point in the same direction. Thus, there are two points $s_{i}<s_{j}$ such that $\Phi(\gamma, v)\left(s_{i}\right)=$ $\Phi(\gamma, v)\left(s_{j}\right)$ and $\theta\left(s_{i}\right)=\theta\left(s_{j}\right) \pm 2 n \pi$ for some integer $n$. Since $\theta$ is monotone and $\gamma$ is simple closed, $n \in\{0,1,-1\}$ by Lemma 2.5. If $n=0$, then $\theta\left(s_{i}\right)=\theta\left(s_{j}\right)$, that is, $\theta$ is constant on the closed interval $\left[s_{i}, s_{j}\right]$. This contradict to $L_{s_{i}} \neq L_{s_{j}}$. If $n= \pm 1$, then $\theta$ is constant on the set $I \backslash\left[s_{i}, s_{j}\right]$. This contradicts $L_{s_{i}} \neq L_{s_{j}}$. Therefore, the frontal $\gamma$ is convex.

We prove the main theorem as follows.

Proof of Theorem 1.6. By combining Lemmas 2.1 and 2.6, we obtain Theorem 1.6.

\section{Examples} of them.

We show some examples of simple closed frontals and discuss on convexity 
Example 3.1. Consider a closed Legendre curve $(\gamma, v):[0,2 \pi] \rightarrow \mathbf{R}^{2} \times S^{1}$ defined by

$$
\gamma(t)=\left(\cos ^{3} t, \sin ^{3} t\right), \quad v(t)=(\sin t, \cos t) .
$$

Then the frontal $\gamma$ is simple closed. Since $\boldsymbol{\mu}(t)=(-\cos t, \sin t)$, the curvature of the Legendre curve is given by $(\ell(t), \beta(t))=(-1,3 \cos t \sin t)$. The sign of $\beta$ changes around $t=0, \pi / 2, \pi, 3 \pi / 2$ and $2 \pi$. By Theorem 1.6, $\gamma$ is not a convex frontal, see Figure 1.

Example 3.2. Consider a closed Legendre curve $(\gamma, v):[0,2 \pi] \rightarrow \mathbf{R}^{2} \times S^{1}$ defined by

$$
\gamma(t)=\left(\frac{1}{3} \cos ^{3} t, \sin t-\frac{1}{3} \sin ^{3} t\right), \quad v(t)=(\cos t, \sin t) .
$$

Then the frontal $\gamma$ is simple closed. Since $\boldsymbol{\mu}(t)=(-\sin t, \cos t)$, the curvature of the Legendre curve is given by $(\ell(t), \beta(t))=\left(1, \cos ^{2} t\right)$. By Theorem 1.6, $\gamma$ is a convex frontal, see Figure 2 left.

Example 3.3. Consider a closed Legendre curve $(\gamma, v):[0,2 \pi] \rightarrow \mathbf{R}^{2} \times S^{1}$ defined by

$$
\gamma(t)=\left(\frac{1}{3} \cos ^{3} t-\frac{1}{5} \cos ^{5} t, \frac{1}{3} \sin ^{3} t-\frac{1}{5} \sin ^{5} t\right), \quad v(t)=(\cos t, \sin t) .
$$

Then the frontal $\gamma$ is simple closed. Since $\boldsymbol{\mu}(t)=(-\sin t, \cos t)$, the curvature of the Legendre curve is given by $(\ell(t), \beta(t))=\left(1, \cos ^{2} t \sin ^{2} t\right)$. By Theorem 1.6, $\gamma$ is a convex frontal, see Figure 2 center.

Example 3.4. Consider a closed Legendre curve $(\gamma, v):[0, \pi] \rightarrow \mathbf{R}^{2} \times S^{1}$ defined by
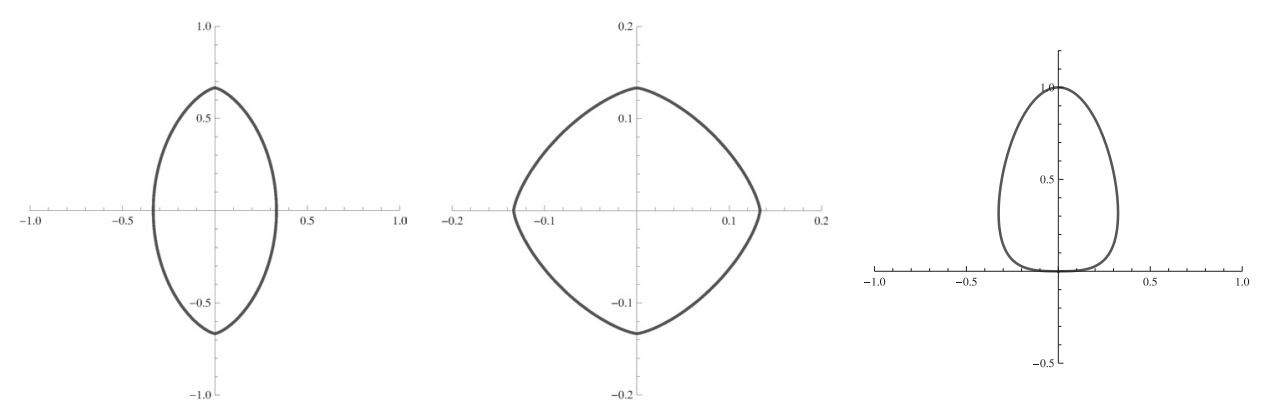

FIGURE 2. Convex simple closed frontals. 


$$
\begin{aligned}
& \gamma(t)=\left(\cos t \sin ^{3} t, \sin ^{8} t\right), \\
& v(t)=\frac{1}{\sqrt{\left(8 \sin ^{5} t \cos t\right)^{2}+\left(3-4 \sin ^{2} t\right)^{2}}}\left(8 \sin ^{5} t \cos t, 4 \sin ^{2} t-3\right) .
\end{aligned}
$$

Then the frontal $\gamma$ is simple closed. Since

$$
\boldsymbol{\mu}(t)=\frac{1}{\sqrt{\left(8 \sin ^{5} t \cos t\right)^{2}+\left(3-4 \sin ^{2} t\right)^{2}}}\left(3-4 \sin ^{2} t, 8 \sin ^{5} t \cos t\right),
$$

the curvature of the Legendre curve is given by

$$
\begin{aligned}
& \ell(t)=\frac{8 \sin ^{4} t\left(16 \sin ^{4} t-30 \sin ^{2} t+15\right)}{\left(8 \sin ^{5} t \cos t\right)^{2}+\left(3-4 \sin ^{2} t\right)^{2}}, \\
& \beta(t)=-\sin ^{2} t \sqrt{\left(8 \sin ^{5} t \cos t\right)^{2}+\left(3-4 \sin ^{2} t\right)^{2}} .
\end{aligned}
$$

Note that $16 \sin ^{4} t-30 \sin ^{2} t+15=\left(4 \sin ^{2} t-15 / 4\right)^{2}+15 / 16>0$. By Theorem 1.6, $\gamma$ is a convex frontal, see Figure 2 right.

\title{
REFERENCES
}

[ 1 ] V. I. ARnOL'D, Singularities of caustics and wave fronts. Mathematics and its applications 62 , Kluwer Academic Publishers, 1990.

[2] V. I. Arnol'D, S. M. Gusein-Zade And A. N. Varchenko, Singularities of differentiable maps I, Birkhäuser, 1986.

[3] T. Fukunaga AND M. TAKAhashi, Existence and uniqueness for Legendre curves, J. Geometry, 104 (2013), 297-307.

[4] C. G. Gibson, Elementary geometry of differentiable curves: An undergraduate introduction, Cambridge University Press, Cambridge, 2001.

[5] A. Gray, E. Abbena and S. Salamon, Modern differential geometry of curves and surfaces with Mathematica, 3rd ed., Studies in advanced mathematics, Chapman and Hall/CRC, Boca Raton, FL, 2006.

\author{
Tomonori Fukunaga \\ KyUShu SANGYo UNIVERSITY \\ FUKUOKA 813-8503 \\ JAPAN \\ E-mail: tfuku@ip.kyusan-u.ac.jp \\ Masatomo Takahashi \\ Muroran Institute of Technology \\ MURORAN 050-8585 \\ JAPAN \\ E-mail: masatomo@mmm.muroran-it.ac.jp
}

